\title{
Éditorial : notes de lecture à propos du cinquième centenaire de la publication des thèses de Martin Luther
}

Gérald Chaix

\section{OpenEdition}

Journals

Édition électronique

URL : http://journals.openedition.org/ifha/9106

DOI : $10.4000 /$ ifha. 9106

ISSN : 2198-8943

Éditeur

IFRA - Institut franco-allemand (sciences historiques et sociales)

Référence électronique

Gérald Chaix, «Éditorial : notes de lecture à propos du cinquième centenaire de la publication des thèses de Martin Luther », Revue de l'IFHA [En ligne], Date de recension, mis en ligne le 14 juin 2018, consulté le 24 septembre 2020. URL : http://journals.openedition.org/ifha/9106 ; DOI : https://doi.org/ 10.4000/ifha.9106

Ce document a été généré automatiquement le 24 septembre 2020.

(CIFHA 


\title{
Éditorial : notes de lecture à propos du cinquième centenaire de la publication des thèses de Martin Luther
}

\author{
Gérald Chaix
}

C'est bien sûr par un concert - Ein feste Burg ist unser Gott - donné dans la Georgenkirche d'Eisenach et par une cérémonie officielle organisée à Wittenberg que s'est achevé, le 31 octobre 2017, le jubilé de la Réforme, unanimement décrété " événement mondial » par le Bundestag le 6 juillet 2011. Cette année du jubilé avait commencé un an plus tôt, en 2016, à Berlin, par la célébration d'un office religieux à la Marienkirche et par une manifestation officielle dans la salle de concert de Gendarmenmarkt. Ainsi, tout au long de la "décennie Luther " (Lutherdekade), débutée en 2008, dignitaires de l'Église évangélique allemande (EKD) et représentants du monde politique ont fait cause commune pour élaborer un programme, fixer un thème pour chacune des dix années, assurer le financement des multiples manifestations et donner à ce projet national un écho mondial. D'emblée, la dimension touristique, liée à l'essor du tourisme culturel, a été présente. Le Land de Saxe-Anhalt a su mobiliser à son profit les subventions pour améliorer ses infrastructures déficientes. De la gare jusqu'au château, la ville de Wittenberg a été profondément rénovée. Devenu une figurine Playmobil, Luther s'est vendu à plus d'un demi-million d'exemplaires !

Jamais, depuis 1617 , la commémoration de la publication des thèses de Luther n'avait eu une telle ampleur. Dès 1817, bien que la mémoire du réformateur fût désormais associée à l'affirmation de la nation allemande, la célébration avait pris une dimension internationale, à l'échelle du protestantisme. Il en avait été de même en 1917, malgré le conflit mondial et le fait que Luther était devenu le héros allemand par excellence, comme l'avait montré le quatrième centenaire de sa naissance en 1883. Le cinquième centenaire ne s'est cependant pas inscrit dans cette tradition. Il a voulu rompre avec la tonalité nationaliste adoptée au cours du XIX siècle et poursuivie jusqu'aux 
lendemains de la deuxième guerre mondiale. Par ailleurs, confrontés à la sécularisation croissante des sociétés européennes, à la désaffection religieuse, particulièrement sensible dans les territoires - Thuringe, Saxe - qui furent ceux du professeur de l'université de Wittenberg, né et mort à Eisleben (1483-1546), formé à l'université d'Erfurt et entré chez les Augustins de cette ville, attentifs en outre aux aspirations œcuméniques, les organisateurs ont voulu éviter les exacerbations confessionnelles.

Enfin, la commémoration de 2017 s'est voulue explicitement historique. Elle s'est attachée à relativiser une héroïsation, déjà à l'œuvre dans les représentations iconographiques et littéraires contemporaines et partie prenante de l'entrée en scène du frère augustin, portraituré dès 1518 par Cranach, occasionnellement représenté sous les traits de l'Hercules germanicus et exalté comme le défenseur des "libertés allemandes ». L'heure du bilan est venue. Les deux acteurs principaux s'y sont risqué (5), suivis déjà par quelques voix discordantes (45). Il mérite une étude approfondie. On ne trouvera ici qu'une simple note critique. Elle a été rédigée sur la base de douze ouvrages parvenus pour compte-rendu (indiqués par un astérisque). Ils sont tous de langue allemande, à une exception près. Cette focalisation sur l'historiographie de langue allemande des dix dernières années (2008-2017) a été conservée dans la sélection de soixante-quatre ouvrages (il y a un $\mathrm{n}^{\circ}$ 35bis) présentée en appendice et servant de mise en perspective.

\section{Survol d'une décennie : Lieux, rythmes, auteurs}

La cartographie des soixante-quatre publications (liste longue), qui ne fait qu'amplifier ce qu'indiquent les lieux d'édition des douze ouvrages (liste courte), rappelle la dispersion des maisons d'édition allemandes sur l'ensemble du territoire et leurs liens souvent étroits avec le réseau universitaire. Elle souligne également l'importance des collections traduisant ces liens et exprimant une ligne éditoriale cohérente. Fondée en 1801, implantée ultérieurement à Tübingen, la maison d'édition Mohr Siebeck en est le meilleur exemple. Elle publie à elle seule quatorze des soixante-quatre livres de la liste longue $(16,17, * 19,20,26,27,56, * 60)$. Adossée à la faculté de théologie et plus précisément à la chaire d'histoire ecclésiastique traitant du Moyen Âge et de la Réformation, la collection "Spätmittelalter, Humanismus und Reformation", dirigée par Volker Leppin, titulaire de la chaire, en accueille six, dont quatre (soulignés) publiés précisément par ce dernier $(1,4,28,35,35 \mathrm{bis}, 39,40)$.

Sans surprise, c'est l'Evangelische Verlagsanstalt de Leipzig qui arrive en deuxième position avec sept publications $(5,9,18,30,32, * 38,48)$. Les collections y sont nombreuses (Arbeiten zur Kirchen-und Theologiegeschichte; Schriften der Stiftung Luthergedenkstätten in Sachsen-Anhalt). Le lien avec le titulaire de la chaire d'histoire de la Réformation Armin Kohnle y est apparent $(9,32)$. L'affirmation d'une identité confessionnelle, liée à l'histoire de la Saxe, à la localisation en zone d'occupation soviétique puis en RDA, à l'actuelle désaffection religieuse, notamment dans ce Land, y est exprimée par exemple par l'ouvrage de Joachim Köhler (30). Mais toutes les maisons d'édition sont présentes : aussi bien le Gütersloher Verlagshaus, éditeur du Verein für Reformationsgeschichte, fondé en 1883 (12), que l'éditeur de Göttingen Vandenhoeck \& Ruprecht (10), mobilisant ses collections $(29,42,57,59)$ et notamment l'emblématique Refo500 Academic Studies, intentionnellement critique et dirigée par Herman Selderhuis, professeur d'histoire ecclésiastique à l'université de théologie d'Apeldorn (lui-même éditeur, en 2015, du dix-septième volume - Reformation und Rationalität (58) - d'une série qui en compte quarante-huit à ce jour), apparue en 2011 à l'occasion justement du 
cinquième centenaire. Les éditeurs réputés « catholiques » ne sont pas restés à l'écart. Soit qu'ils aient choisi d'apporter un éclairage confessionnellement identifié, sans pour autant être conflictuel $\left({ }^{*} 2\right.$, Paderborn: Schöning), soit qu'ils aient privilégié une approche explicitement œcuménique $(6,15,21$, Freiburg: Herder), se traduisant éventuellement par une coopération éditoriale (42, Freiburg-Göttingen : Herder-V\&R).

Quantitativement, les éditeurs "profanes" sont nettement en retrait. Leur rôle est cependant déterminant. S'ils sacrifient parfois à la nostalgie d'un récit traditionnel (Berlin : Rowohlt, 63), ce sont eux qui publient (ou traduisent) les biographies qui ont marqué la commémoration (Berlin : Suhrkamp, 21 ; Stuttgart : Reclam, ${ }^{*} 31$; Francfort : Fischer, 51) et les histoires générales de la Réformation inscrivant la Réforme luthérienne dans le temps long des Réformes, dans l'espace européen et dans une interprétation globale (Berlin : Suhrkamp, 25). Ce sont aussi leurs publications qui ont connu le plus large succès, à en juger par les rééditions. L'éditeur munichois C.H. Beck est à cet égard exemplaire. Les deux titres de son excellente collection "Beck Wissen ", directement inspirée des "Que sais-je? ", consacrés respectivement à l'histoire de la Réforme (55, 1996 $\rightarrow 2017$; trad. italienne) et à la biographie de Luther $(24,2006 \rightarrow 2017$; trad. anglaise, espagnole, italienne), publiés avant le début de la décennie, en ont accompagné le déploiement. La biographie du réformateur demandée à Heinz Schilling et publiée dès 2012 s'est nettement imposée $\left({ }^{*} 53,4^{\mathrm{e}}\right.$ éd. en 2016 ; trad. anglaise, danoise, française, italienne, russe, slovaque). Il en est de même, dans une moindre mesure, pour l'histoire de la Réformation due à Thomas Kaufmann, publiée en 2016 et rééditée l'année suivante $\left({ }^{*} 22\right)$. Sans oublier les approches originales que l'éditeur a également proposées : «1517: histoire mondiale d'une année » $\left({ }^{*} 52\right)$, « les racines mystiques » de Luther (*37), « Rome et la Réformation » $\left.*^{*} 50\right)$.

La progressive montée en puissance du rythme des publications est évidente. La publication du Luther de Schilling (*53) fait office de prélude. Le nombre des publications augmente d'année en année $(2013: 5 ; 2014: 8 ; 2015: 10)$ et culmine en 2016 (17) et 2017 (17). D’emblée, les ténors rappellent les enjeux : « À qui appartient la Réformation? » $(15,2013)$ ", l'historicisation $(54,2014)$, la perspective œcuménique (42, 2014), la dimension européenne $(11,2015)$. À partir de 2015, chaque auteur avance ses pions $(* 31,32,56)$, les instruments de travail sont mis à disposition $(34,41)$. Le temps est alors venu de la publication des colloques, des essais et des monographies, des numéros spéciaux de revues, de la présentation des expositions et de leurs catalogues, des articles de magazines et de journaux, des interventions radiophoniques et télévisées, des concerts et des prédications.

Apparemment sans grande valeur statistique, la «liste courte " des dix auteurs est étonnamment représentative. Les auteurs sont très majoritairement des hommes : 8 sur 10 (80\%). La « liste longue » confirme ce pourcentage : 41 sur 52 ( $75,7 \%)$. Il en va de même si l'on prend en compte les 340 auteurs mis à contribution dans les ouvrages collectifs et s'ajoutant aux 52 noms précédents: 278 sur 340 (77,7\%). Tous universitaires, ils ont, à une exception près $\left({ }^{*} 2\right)$, plus de 50 ans et sont titulaires d'une chaire (trois sont cependant émérites : ${ }^{*} 19,{ }^{*} 31,{ }^{*} 52$ et ${ }^{*} 53$ ). La « liste longue » n'est guère différente : elle accueille peu d'auteurs ou d'éditeurs de moins de 40 ans $(1,4,18)$, augmente le nombre des émérites (voire, par le jeu d'une réédition, fait place à Heiko Oberman, aujourd'hui décédé, dont la biographie de Luther avait marqué la commémoration de 1983,47), introduit quelques francs-tireurs $(30,61,63)$, et confirme finalement la prépondérance des « pontes ». 
Restent deux particularités, liées à l'évidence à l'objet de la commémoration mais qui en rappellent précisément la place, nullement figée ni uniformément identique, dans les champs disciplinaires scientifiques ainsi que la dimension identitaire. Cinq des dix auteurs de la « liste courte » enseignent l'histoire ecclésiastique (Kirchengeschichte) dans une faculté de théologie, trois dépendent d'un département d'histoire, l'un est juriste et le dixième enseigne la «médiologie " (Medienwissenschaft). La " liste longue » confirme cette particularité (seize Kirchenhistoriker) en y ajoutant même huit enseignants en faculté de théologie. La situation institutionnelle des 340 auteurs d'article montre que cette particularité est très largement répandue et n'est pas propre à l'Allemagne. On note sans surprise que les deux seuls auteurs français présents dans ce corpus enseignent à la faculté de théologie protestante de Strasbourg, l'un l'histoire ecclésiastique, l'autre la théologie systématique. La deuxième particularité, liée à la précédente, est le rappel institutionnel de l'identité confessionnelle: la plupart des Kirchenhistoriker sont ainsi rattachés à une faculté de théologie évangélique et il en va de même pour les théologiens, à une exception près (Jan-Heiner Tück, 6). Il n'est d'ailleurs pas rare qu'ils aient une responsabilité ecclésiale et qu'ils exercent une tâche pastorale.

\section{L'arrière-plan d'une commémoration : expositions, éditions, instruments de travail}

Des multiples manifestations - conférences, colloques, cours, concerts et enregistrements, séries télévisées, films, expositions...- qui ont scandé la commémoration et "transmis » Luther (Luther vermitteln, 18), on ne retiendra, pour leur permanente utilité, que les catalogues des expositions les plus significatives, les éditions de textes et quelques instruments de travail indispensables. Quatre expositions ont bénéficié d'une labellisation nationale :

- Torgau: "Luther und die Fürsten. Selbstdarstellung und Selbstverständnis des Herrschers im Zeitalter der Reformation ", 2 vol. (catalogue + actes du colloque), Dresde : Sandstein, 2015, 360-348 p. (Claudia Brink : 18, 148-163).

-Wartburg: "Luther und die Deutschen. Wie jede Epoche ihr eigenes Lutherbild prägte", Petersberg : Michael Imhof, 2017, 457 p. (Marc Höchner: 18, 173-183).

-Wittenberg : « Luther! 95 Schätze - 95 Menschen. Luthers greibares Erbe », Munich : Hirmer, 2017, 623 p. (Mirko Gutjahr: 18, 184-192).

- Berlin : «Der Luther Effekt. 500 Jahre Protestantismus in der Welt », Munich : Hirmer, 432 p.

Placées sous une commune appellation «Here I stand », trois grandes expositions ont été organisées aux États-Unis (Minneapolis : "Art and the Reformation »; New York : "Word and Image: Martin Luther's Reformation »; Atlanta: "Law and Grace, Lucas Cranach, and the Promise of Salvation »). Elles ont suscité deux importants volumes, signalés ici dans leur version allemande, publiée à Dresde chez Sandstein en 2016: Martin Luther. Aufbruch in eine neue Welt (495 p.); Martin Luther. Schätze der Reformation (503 p.). Toutes trois soulignent l'importance de Lucas Cranach et de son atelier (voir aussi, à ce sujet, les contributions de Anja Ottilie Ilg, 21, 161-198; Perdita Ladwig, 13, 181-202; Ruth Slenczka 11, 81-102, 54, 133-158).

Les expositions organisées en Allemagne ont souvent mis en évidence le retentissement de la réforme de Wittenberg et les spécificités régionales d'un éventuel écho: Braunschweig ( $I m$ Aufbruch Reformation, 1517-1617», Dresde : Sandstein, 2017, 456 p.), Gottorf et Greifswald («Luthers Norden», Petersberg: Michael Imhof, 2017, 320 p.), Neuburg a. d. Donau («Fürsten Macht und wahrer Glaube - Reformation und 
Gegenreformation. Das Beispiel Pfalz-Neuburg ", Regensburg Friedrich Pustet, 2017, 452 p.), Ostfildern ("Freiheit - Wahrheit - Evangelium. Reformation im Württemberg», 2 vol. (catalogue + contributions, Ostfildern: Jan Thorbecke, 2017, $416+336$ p.), Potsdam ( "Reformation und Freiheit. Luther und die Folgen für Preußen und Brandenburg", Petersberg: Michael Imhof, 2017, 304 p.). Tirant éventuellement parti des ressources locales, elles ont mis en valeur tel ou tel aspect : Nuremberg et le monde en mutation au XVI ${ }^{\mathrm{e}}$ siècle («Luther, Kolumbus und die Folgen? 1500-1600», Nuremberg: Verlag des Germanischen Nationalmuseums, 2017, 312 p.), Rothenburg et les sorcières (« Mit dem Schwert oder festem Glaube. Luther und die Hexen », Rothenburg: Theiss, 2017, 224 p.), Veste Coburg, les chevaliers et les paysans ("Ritter, Bauern, Lutheraner », Darmstadt: Theiss, 2017, 392 p.), Zeitz et le dialogue des confessions («Dialog der Konfessionen. Bischof Julius Pflug und die Reformation ", Schriftenreihe der Vereinigten Domstifter zu Merseburg und Naumburg und Kollegialstifts Zeitz, 10, Petersberg: Michael Imhof, 2017,528 p.).

L'édition scientifique des $F u v r e s$ de Luther avait emblématiquement débuté à Weimar à l'occasion de la commémoration de 1883. Cette monumentale entreprise s'est achevée au début de la présente décennie, en 2009. Au total, conservant le format in-4, 123 volumes ont été publiés, totalisant quelque 80000 pages. L'édition (WA) comprend quatre ensembles: les "propos de table" (Tischreden, TR, 6 vol.), la Bible allemande (Deutsche Bibel, DB, 15 vol.), la correspondance (Briefwechsel, BR, 18 vol.) et les écrits (Schriften/Werke, 84 vol.). Elle est pourvue d'index, est disponible en CD-Rom et forme une base de données accessible sous conditions par internet. Elle se prolonge par la série « Archiv zur Weimarer Aufgabe der Werke Martin Luthers ». Inaugurée en 1984, celle-ci compte neuf volumes à ce jour.

La présente commémoration a été l'occasion de republier, isolément ou dans des anthologies, généralement assorties de commentaires, les œuvres les plus importantes de Luther, tel l'appel À la noblesse chrétienne de la nation allemande présenté par Thomas Kaufmann et publié dans la collection dirigée par lui chez l'éditeur Mohr Siebeck (26). Ce souci de contextualisation vaut aussi bien pour les " propos de table » (1) que pour les écrits du «jeune Luther»(12). Il aide à la lecture des textes polémiques et notamment les écrits de Luther sur les Juifs $(23,27)$ ainsi que sur les Turcs, fidèles de la religion musulmane et adversaires de l'Empire et des possessions habsbourgeoises (29).

Plus encore que l'utile rassemblement d'articles jalonnant les recherches sur Luther au cours des quarante dernières années (7), trois instruments de travail méritent d'être signalés. Tout d'abord, en langue anglaise mais avec une participation internationale (et germanophone) importante, l'oxford Handbook of Martin Luther's theology et ses quarante-sept entrées rédigées par les meilleurs spécialistes et couvrant l'ensemble de la problématique théologique (34). Ensuite, réédité dès l'année suivante malgré son prix élevé, le Luther-Lexikon (41): les 586 articles en sont bien plus concis, mais les bibliographies sont à peine plus réduites et tous les angles d'attaque sont pris en considération: les œuvres et les concepts, les éléments biographiques et les personnages contemporains, les réalités historiques et les institutions, la réception de Luther dans le temps et dans l'espace (jusqu'à nos jours) ainsi que les grandes figures de l'historiographie des XIX ${ }^{\mathrm{e}}$ et $\mathrm{XX}^{\mathrm{e}}$ siècles. Enfin, pour l'ampleur d'une entreprise voulant embrasser tous les aspects du chrétien Martin Luther " entre Réformes et modernité (1517-2017) », la «somme » rassemblée par Alberto Melloni, professeur d'histoire du 
christianisme à l'université de Modena-Reggio Emilia, est disponible en trois volumes aussi bien en langue allemande qu'en langue anglaise (46).

Luther mis en perspective : réformes et Réformation, l'année 1517, Moyen Âge et Modernité

La volonté d'historiciser Luther, de replacer la Réforme de Wittenberg à la fois dans une durée plus longue que celle de la vie du réformateur (10) et dans un espace plus large que celui du Saint Empire (11), mais aussi de prendre en compte la globalité des mutations qui s'opèrent entre les $\mathrm{XV}^{\mathrm{e}}$ et $\mathrm{XVI}^{\mathrm{e}}$ siècles, sous-tend les histoires de la Réformation qui ont été publiées, sous une forme resserrée par Volker Leppin $(36,44)$, de manière plus prolixe par Thomas Kaufmann (25).

Aujourd'hui émérite mais toujours très actif, Heinz Schilling avait pris une longueur d'avance en publiant dès 2012 sa monumentale biographie de Luther (*53). C'est en se concentrant sur l'année 2017, replacée dans une perspective mondiale, qu'il opère la mise en perspective de la publication des 95 thèses, le 31 octobre.

Un siècle après l'exaltation, en 1883, d'un Luther devenu le héros allemand par excellence, le précurseur de la modernité, le parangon des vertus bourgeoises, Heiko Oberman, alors professeur d'histoire ecclésiastique à l'université de Tübingen et directeur de l'Institut du Moyen Âge tardif et de la Réformation, avait marqué la commémoration de 1983 en publiant, un an auparavant, sa biographie de Luther, « un homme entre Dieu et Diable». Elle demeure un ouvrage de référence et a été logiquement rééditée en 2016 (47). Le réexamen du lien entre Moyen Âge, Réformation et Modernité, au cœur de l'historiographie allemande depuis le XIX ${ }^{\mathrm{e}}$ siècle, a été l'un des fils conducteurs de la décennie (14). Il inspire largement la réflexion de Berndt Hamm (16), longtemps titulaire de la chaire d'histoire ecclésiastique à l'université d'Erlangen, aujourd'hui émérite, et de Volker Leppin, successeur d'Oberman à Tübingen (40). Il explique l'intérêt général porté au « jeune Luther » et aux débuts du mouvement réformateur $(4,17,28,39,40)$ et, dans de nombreuses contributions de V. Leppin, l'attention accordée aux « racines mystiques » du théologien de Wittenberg $\left(34,49-61 ;{ }^{*} 37 ; 46,157-172\right)$ va à rebours de l'historiographie protestante jusqu'à une date récente (21, 269-292: «Mystik und Neuzeit. Die Luther Interpretationen der Holl-Schule in der theologischen Debatten der Weimarer Republik »).

Pleins feux sur Luther : « homme de Dieu ", « renégat ", « hérétique "

Les biographies de Luther n'ont jamais manqué. La décennie a été l'occasion de republier soit celles qui demeurent significatives (47: Oberman), soit celles qui appartiennent à des collections de référence (24: Kaufmann (Beck Wissen), 35bis : Leppin (Gestalten des Mittelalters und der Renaissance), 57 : Schwarz (Die Kirche in ihrer Geschichte). Elle a donné la possibilité à des auteurs aux marges des institutions universitaires de redonner vie aux interprétations héroïsantes $(30,63)$, mais aussi aux spécialistes de proposer leur vision, tantôt sous une forme brève (32: Kohnle, 43 : Leppin), tantôt de manière plus détaillée ( $*_{51}$ : Roper, $*_{53}$ : Schilling). Omniprésent, Volker Leppin est, avec Dietrich Korsch, l'un des deux éditeurs de l'ouvrage collectif consacré à l'étude du lien entre la biographie et la réflexion théologique de Luther (35). Classique, Ulrich Köpf a sobrement présenté l'« homme de Dieu ».

Australienne, Lyndal Roper enseigne à l'université d'Oxford. Ses premières recherches sur la sorcellerie dans l'espace impérial l'avait fait connaitre pour son approche tout à la fois anthropologique et psycho-analytique. Publiée en anglais mais aujourd'hui traduite en allemand, sa biographie sur Luther, renégat et prophète, s'inscrit dans cette 
veine. Elle est aussi le fruit de dix années de travail, dont les premiers résultats avaient paru en 2010.

Plus qu'un aboutissement, la condamnation de Luther pour hérésie est un élément qui l'oblige à préciser sa pensée et le pousse à prendre position face à une papauté où s'entremêlent stratégies familiales, calculs politiques, ambitions culturelles, redéfinition du pouvoir pontifical et besoins financiers (33, 113-126: Armin Kohnle, " Die Päpste; Von der Renaissance zur Gegenreformation »). Le rôle de la Curie (33 ; 54, 3-30 : Götz Rüdiger Tewes, "Die Kurie unter dem Medici-Papst Leo X. und die Phase der beginnenden Reformation Luthers: familiäre Interessen statt universaler Pflichten »), mais aussi celui des adversaires de Luther (62), ont ainsi fait l'objet d'une attention particulière.

\section{Luther au regard des théologiens, des juristes et des historiens des idées politiques}

L'analyse du corpus des auteurs a mis en évidence, contrastant avec la situation française, l'importance des théologiens et des historiens de l'Église, les deux compétences étant souvent réunies dans une même personne. La théologie de Luther a fait l'objet d'une solide mise au point par Reinhard Schwarz, professeur émérite d'histoire ecclésiastique à l'université de Munich (56). Une autre voie a été suivie par Volker Leppin qui a choisi de rassembler vingt-quatre articles. Effleuré par Volker Leppin ou par Luise Schorn-Schütte (46, 565-574: «Luther and politics»), l'apport de Luther et, plus largement, du protestantisme à l'histoire du droit et des idées politiques a fait l'objet d'une monumentale mise au point par l'historien du droit Martin Heckel, né en 1929, professeur émérite à l'université de Tübingen.

Luther pour aujourd'hui : "retour à Luther", "perspectives œcuméniques", Luther historique

L'importance accordée à l'historicisation de Luther s'est assez logiquement reflétée dans les livres qui ont été reçus pour compte-rendu et dans la sélection retenue de soixante-quatre ouvrages. Le "retour à Luther » est cependant le titre explicite d'une des douze recensions.

L'un des fils conducteurs de la décennie a été le rôle que la commémoration pouvait jouer dans le dialogue œcuménique $(6 ; 8 ; 21 ; 42 ; 46,843-860$ : Sarah Hinlicky Wilson, «Lutheranism in Ecumenical Dialogue »; 861-882, Enrico Cavalotti : « From Excommunicated to Common Teacher. Luther and the Ecumenical Movement »; 59, 129-152 , Ulrike Schuler: « Was tun mit 2017? Die ökumenische Herausforderung des Jubiläums aus methodischer Perspektive »). De manière plus large encore, revenant sur l'attitude de Luther à l'égard des Juifs (6, 428-442, Hartmut Lehmann : « Luther und die Juden. Stolpersteine auf dem Weg zur Fünhundertjahrfeier der Reformation $2017 » ; 23,27,35,251-270$, Anselm Schubert : « Fremde Sünde. Zur Theologie von Luthers späten Judenschriften»; 46, 617-634, Brooks Schramm : "Luther and the Jews ») et des Musulmans (6, 443-469, Karl-Josef Kuschel : " Martin Luther, die Türken und der Islam. Ein schwieriges Erbe als Auftrag für heute »; $29 ; 46$, 649-662, Gregory J. Miller: "Luther and the Turks »), l'attention s'est également portée sur le dialogue interreligieux (15, 287-300, Andreas Mühling: «Reformationsgedenken und interreligiöser Dialog»; 34, 435-448, J. Paul Rajashekar: "Luther as a Resource for Christian Dialogue with Other World Religions »).

Dans le cadre de ces "notes de lecture ", c'est bien cependant le "Luther historique " qui s'impose comme l'axe majeur de la décennie écoulée, comme en témoigne d'ailleurs 
le succès des biographies, qu'elles soient volontairement réduites (24) ou, comme celle due à Heinz Schilling, naturellement volumineuses.

Le volume inhabituel de ces « notes de lecture » reflète l'ampleur, voire pour certains la démesure, de la « décennie Luther ». Encore n'est-il ici question, sauf exceptions, que de la production publiée en Allemagne et retenue par les éditeurs. Même limitée à cette sélection, la prise en compte de toutes les contributions présentées dans les ouvrages collectifs permettrait d'ailleurs une présentation plus détaillée des sources et de l'historiographie, des liens entre biographie et production théologique, des influences immédiates ou différées, brutales ou imperceptibles, diverses et complexes - sur les évolutions religieuses et culturelles, sur les institutions politiques et sociales, sur les comportements individuels et collectifs.

Dressé immédiatement au terme de cette décennie, ce premier bilan semble indiquer que les objectifs fixés par les organisateurs - Églises, État et collectivités politiques - et largement partagés par la communauté scientifique et les institutions culturelles ont été globalement atteints: récuser l'interprétation étroitement nationale, voire franchement nationaliste, des historiographies des deux siècles antérieurs, devenues elles-mêmes objet d'étude, replacer pour les uns l'affirmation confessionnelle dans une perspective œcuménique, proposer pour les autres une lecture globalisante du religieux, pour tous restituer l'environnement dans lequel évoluait Luther, contextualiser ses paroles, ses écrits et ses comportements, intégrer la Réforme luthérienne dans un mouvement plus vaste dans le temps et dans l'espace sans pour autant nier sa singularité et son exemplarité, elles-mêmes questions pour l'historien.

La production historiographique - mais aussi théologique - qui a accompagné et scandé la « décennie Luther » témoigne de la vitalité des institutions - religieuses, politiques, scientifiques - qui en ont été les artisans, et de l'intérêt, multiforme et d'intensité variable, qu'y ont trouvé touristes et pèlerins, auditeurs et lecteurs, spécialistes et amateurs. Elle en révèle les constantes et les évolutions, les spécificités nationales et les impulsions internationales. Dans un monde étranger à bien des égards à celui dans lequel vivait Luther, elle souligne l'intérêt que celui-ci continue d'exercer, moins comme héros d'une nation ou d'une Église que comme sujet d'une histoire critique, plurielle, discutable et partagée. À taille humaine.

Une décennie de publications (2008-2017)

(l'astérisque indique un ouvrage recensé)

1 Katharina Bärenfanger, Volker Leppin (dir.), Martin Luthers Tischreden: Neuansätze der Forschung, Tübingen: Mohr Siebeck (Spätmittelalter, Humanismus, Reformation 71), 2013, VIII-263 p., $89 €$

2 *Daniela Blum, Der katholische Luther. Begegnungen-Prägungen-Rezeptionen, Paderborn: Ferdinand Schöningh, 2016, 221 p., 24,90€

3 *Norbert Bolz, Zurück zu Luther, Paderborn: Wilhelm Finck, 2016, 141 p., 19,90€

4 Christoph Burger, Tradition und Neubeginn: Martin Luther in seinen frühen Jahren, Tübingen: Mohr Siebeck (Spätmittelalter, Humanismus und Reformation 79), 2014, XIV-251 p. [recueil d'articles], $89 €$

5 Johann Heinrich Claussen, Stefan Rhein (dir.), Reformation 2017. Eine Bilanz, Leipzig: Evangelische Verlagsanstalt, 2017, 192 p., $24 €$

6 Christian Danz, Jan-Heiner Tück (dir.), Martin Luther im Widerstreit der Konfessionen. Historische und theologische Perspektiven, Freiburg/B.: Herder, 2017, 531 p., 24,99€ 
7 Christian Danz (ed.), Martin Luther, Darmstadt, Wissenschaftliche Buchgesellschaft (Neue Wege der Forschung), 2015, 229 p. [reprise d'articles parus entre 1978 (Reinhard Schwarz) et 2010 (Berndt Hamm)], 39,95€

8 Mariano Delgado, Volker Leppin (dir.), Luther: Zankapfel zwischen den Konfessionen und "Vater im Glauben". Historische, systematische und ökumenische Zugänge, FribourgStuttgart: Academic Press-W. Kolhammer (Studien zur christlichen Religionsgeschichte 21), 2016, 422 p., $69 €$

9 Irene Dingel, Armin Kohnle, Stefan Rhein, Ernst-Joachim Waschke (dir.), Initia Reformationis. Wittenberg und die frühe Reformation, Leipzig: Verlagsanstalt (Leucorea Studien zur Geschichte der Reformation und der Lutherischen Orthodoxie 33), 2017, 444 p., $68 €$

10 Irene Dingel, Reformation. Zentren-Akteure-Ereignisse, Göttingen: Vandenhoeck \& Ruprecht, 2016, 308 p., 32,99€

11 Irene Dingel, Ute Lotz-Heumann, (dir.), Entfaltung und zeitgenössische Wirkung der Reformation im europäischen Kontext, Gütersloh: Gütersloher Verlagshaus (Schriften des Vereins für Reformationsgeschichte 216), 2015, 388 p., $49 €$

12 Irene Dingel, Hennig P. Jürgens (dir.), Meilensteine der Reformation. Schlüsseldokumente der frühen Wirksamkeit Martin Luthers, Gütersloh: Gütersloher Verlagshaus, 2014, 296 p., $19,99 €$

13 Richard Faber, Uwe Puschner (dir.), Luther. Zeitgenössisch, historisch, kontrovers, Frankfurt: Peter Lang, 2017 (Zivilisationen und Geschichte 50), 771 p., 74,70€

14 Günter Frank, Volker Leppin (dir.), Die Reformation und ihr Mittelalter - Wertung, Wirkung, Perspektiven, Stuttgart-Bad Cannstatt: frommann-holzboog Verlag, 2016, VII-478 p., $68 €$

15 Günter Frank, Volker Leppin, Herman J. Selderhuis (dir.), Wem gehört die Reformation? Nationale und konfessionelle Dispositionen der Reformationsdeutung, Freiburg/B.: Herder, 2013, 304 p., $18 €$

16 Berndt Hamm, Ablass und Reformation - erstaunliche Kohärenzen, Tübingen: Mohr Siebeck, 2016, XVI-281 p., $24 €$

17 Berndt Hamm, Der frühe Luther. Etappen reformatorischer Neuorientierung, Tübingen: Mohr Siebeck, 2010, IX-318 p., $29 €$

18 Benjamin Hasselhorn (dir.), Luther vermitteln. Reformationsgeschichte zwischen Historisierung und Aktualisierung, Leipzig: Evangelische Verlagsanstalt, 2016, 256 p., $24,80 €$

19 * Martin Heckel, Martin Luthers Reformation und das Recht. Die Entwicklung der Theologie Luthers und ihre Auswirkung auf das Recht unter den Rahmenbedingungen der Reichsreform und der Territorialstaatsbildung im Kampf mit Rom und den "Schwärmen", Tübingen: Mohr Siebeck (Jus ecclesiasticum 114), 2016, 988 p., $69 €$

20 Ulrich Heckel, Jürgen Kampman, Volker Leppin, Christoph Schwöbel (dir.), Luther heute: Austrahlungen der Wittenberger Reformation, Tübingen: Mohr Siebeck (UTB 4792), 2017, IX-394 p., 19,99€

21 Andreas Holzem, Volker Leppin, Claus Arnold, Norbert Haag (dir.), Martin Luther: Monument, Ketzer, Mensch. Lutherbilder, Lutherprojektionen und ein ökumenischer Luther, Freiburg/B.: Herder, 2017, 464 p., 34,50€ 
22 *Thomas Kaufmann, Erlöste und verdammte. Eine Geschichte der Reformation, München: C.H. Beck, $2017^{4}$ (2016), 508 p., 26,95€

23 Thomas Kaufmann, Luthers Juden, Stuttgart: Reclam, 2017, 203 p., 22,95€ (trad. française, 2016)

24 Thomas Kaufmann, Martin Luther, München: Beck (Beck Wissen 2388), 2017 (2006), 128 p., $8,95 €$

25 Thomas Kaufmann, Geschichte der Reformation in Deutschland, nouv. éd., Berlin: Suhrkamp, 2016 (2009), 1038 p., $28 €$ (trad. française, 2014)

26 Thomas Kaufmann, An den christlichen Adel deutscher Nation von den christlichen Standes Besserung, Tübingen: Mohr Siebeck (Kommentare zu Schriften Luthers 3), 2014, XV-559 p., $59 €$

27 Thomas Kaufmann, Luthers "Judenschriften". Ein Beitrag zu ihrer historischen Kontextualisierung, Tübingen: Mohr Siebeck, 2013 (2011), XI-231 p., $29 €$

28 Thomas Kaufmann, Der Anfang der Reformation: Studien zur Kontextualität der Theologie, Publizistik und Inszenierung Luthers und der reformatorischen Bewegung, Tübingen: Mohr Siebeck (Spätmittealter, Humanismus, Reformation 67), 2012, XVIII-676 p., $139 €$

29 Thomas Kaufman, "Türckenbüchlein": zur christlichen Wahrnehmung "türkischer Religion" in Spätmittelalter und Reformation, Göttingen: Vandenhoeck \& Ruprecht (Forschungen zur Kirchen- und Dogmengeschichte 97), 2008, 299 p., $80 €$

30 Joachim Köhler, Luther! Biographie eines befreiten, Leipzig: Evangelische Verlagsanstalt, 2017 (2016), 408 p., 22,90€

31 *Ulrich Köpf, Martin Luther. Der Reformator und sein Werk, Stuttgart: Reclam, 2015, 254 p., 22,95€

32 Armin Kohnle, Martin Luther: Reformator, Ketzer, Ehemann, Leipzig: Evangelische Verlagsanstalt, 2015, 223 p., 29,95€

33 Armin Kohnle, Zwischen Reform und Abgrenzung. Die Römische Kurie und die Reformation, Stuttgart: Franz Steiner (Quellen und Forschungen zur sächsischen Geschichte 31), 2014, 248 p., $49 €$

34 Robert Kolb, Irene Dingel, L'Ubomir Batka (dir.), The Oxford handbook of Martin Luther's theology, Oxford: Oxford University Press, 2014, XVII-662 p., 49,98 \$

35 Dietrich Korsch, Volker Leppin (dit.), Martin Luther. Biographie und Theologie, Tübingen: Mohr Siebeck (Spätmittelalter, Humanismus, Reformation 53), 2017 (2010), $\mathrm{X}-335$ p., $59 €$

35bis Volker Leppin, Martin Luther, Darmstadt: Philipp von Zabern (Gestalten des Mittelalters und der Renaissance), 2017 (2006, Darmstadt: Wissenschaftliche Buchgesellschaft), 426 p., 39,95€

36 Volker Leppin, Die Reformation, Darmstadt: Wissenschaftliche Buchgesellschaft, 2017 (2013), 168 p., 19,95€

37 *Volker Leppin, Die fremde Reformation: Luthers mystische Wurzeln, München: C.H. Beck, 2016, 247 p., 21,95€

38 *Volker Leppin, Reformatorische Gestaltungen. Theologie und Kirchenpolitik in Spätmittelalter und Früher Neuzeit, Leipzig: Evangelische Verlagsanstalt (Arbeiten zur Kirchen- und Theologiegeschichte 43), 2016, 392 p., $68 €$ 
39 Volker Leppin (dir.), Reformatorische Theologie und Autoritäten: Studien zur Genese des Schriftprinzips beim jungen Luther, Tübingen: Mohr Siebeck (Spätmittelalter, Humanismus, Reformation 85), 2015, VIII-305 p., $99 €$

40 Volker Leppin, Transformationen: Studien zu den Wandlungsprozessen in Theologie und Frömmigkeit zwischen Spätmittealter und Reformation, Tübingen: Mohr Siebeck (Spätmittelalter, Humanismus, Reformation 86), 2015, XV-559 p., $129 €$

41 Volker Leppin, Gury Schneider-Ludorff (dir.), Das Luther-Lexikon, Regensburg: Bückle \& Böhm, 2015 (2014), 820 p., $98 €$

42 Volker Leppin, Dorothea Sattler, Reformation 1517-2017. Ökumenische Perspektiven, Freiburg-Göttingen: Herder-Vandenhoeck \& Ruprecht (Dialog der Kirchen 16), 2014, 119 p., $22 €$

43 Volker Leppin, Vom Mönch zum Feind des Papstes. Biographie, Darmstadt: Lambert Schneider, 2013, 156 p., 15,99€

44 Volker Leppin, Das Zeitalter der Reformation. Eine Welt im Übergang, Darmstadt: Wissenschaftliche Buchgesellschaft, 2009, 160 p.

45 Christoph Markschies, Aufbruch oder Katerstimmung? Zur Lage nach dem Reformationsjubiläum, Hamburg: Kreuz Verlag, 2017, 128 p., $16 €$

46 Alberto Melloni (dir.), Ein Christ zwischen Reformen und Modern (1517-2017), 3 vol., Berlin-Boston: de Gruyter, 2017, XXXII-1757 p. (A Christian between Reforms and Modernity, 3 vol., Berlin-Boston: de Gruyter, 2017, XXXVI-1720 p.), $399 €$

47 Heiko Oberman, Luther. Mensch zwischen Gott und Teufel, München: Pantheon, 2016 (1982), 447 p., 15,50€

48 Joachim Ott, Martin Treu (dir.), Luthers Thesenanschlag. Faktum oder Fiktion, Leipzig: Evangelische Verlagsanstalt (Schriften der Stiftung Luthergedenkstätten in SachsenAnhalt 9), 2008, 208 p., $36 €$

49 Andreas Rehberg, (dir.), Ablasskampagnen des Spätmittelalters. Luthersthesen von $1517 \mathrm{im}$ Kontext, Berlin-Boston: de Gruyter (Bibliothek des deutschen Historischen Insituts in Rom 132), 2017, XVIII-712 p., 129,95€

50 *Volker Reinhardt, Luther der Ketzer. Rom und die Reformation, München: C.H. Beck, 2016, 352 p., 24,95€

51 *Lyndal Roper, Martin Luther. Renagade and Prophet, London: The Bodley Head, 2016, XIV-577 p., 27,99€ (trad. allemande, Der Mensch Martin Luther, Frankfurt: S. Fischer Verlag, 2016, 736 p., $28 €)$

52 *Heinz Schilling, 1517. Weltgeschichte eines Jahres, München: C.H. Beck, 2017, 364 p., $24,95 €$

53 *Heinz Schilling, Martin Luther. Rebell in einer Zeit des Umbruchs. Eine Biographie, München: C.H. Beck, 2016 (2012), 728 p., 19,95€ (trad. française, 2014)

54 Heinz Schilling (dir.), Der Reformator Martin Luther 2017: eine wissenschaftliche und gedenkpolitische Bestandaufnahme, Berlin: de Gruyter Oldenbourg (Schriften des Historischen Kollegs/Kolloquien 92), 2014, XVII-309 p., 69,95€

55 Luise Schorn-Schütte, Die Reformation. Vorgeschichte, Verlauf, Wirkung, München: Beck (Beck Wissen 2054), 2017 (1996), 128 p., 8,95€

56 Reinhard Schwarz, Martin Luther. Lehrer der christlichen Religion, Tübingen: Mohr Siebeck, 2016 (2015), XIV-544 p., $39 €$. 
57 Reinhard Schwarz, Luther, Göttingen: Vandenhoeck \& Ruprecht (UTB 1926), 2014 (1986), 271 p., 25,99€

58 Herman J. Selderhuis, Ernst-Joachim Waschke (dir.), Reformation und Rationalität, Göttingen, Vandenhoeck \& Ruprecht (Refo500 Academic Studies 17), 2015, 317 p., $55 €$

59 Volker Spangenberg (dir.), Luther und die Reformation aus freikirchlicher Sicht, Göttingen: Vandenhoeck \& Ruprecht unipress, 2013, 198 p., $50 €$

$60{ }^{*}$ Christoph Strohm (dir.), Reformation und Recht. Ein Beitrag zur Kontroverse um die Kulturwirkungen der Reformation, Tübingen, Mohr Siebeck, 2017, IX-219 p., $14 €$

61 Wolfgang Trautwein, Ulrike Horstenkamp, Gabriele Weidle (dir.), Folgenreich. Reformation und Kulturgeschichte, Bonn: Arbeitskreis selbstständiger Kultur-Institute, 2016, 304 p., 19,80€

62 Klaus Unterburger, Unter dem Gegensatz verborgen: Tradition und Innovation in der Auseinandersetzung des jungen Martin Luther mit seinen theologischen Gegnern, Münster: Aschendorff (Katholisches Leben und Kirchenreform im Zeitalter der 62 Glaubensspaltung 74), 2015, 160 p., 24,80€

63 Willi Winkler, Luther. Ein deutscher Rebell, Berlin: Rowohlt, 2016, 640 p., 29,95€

\section{INDEX}

Index chronologique : Période moderne

Thèmes : Histoire religieuse ; Histoire des mentalités

\section{AUTEUR \\ GÉRALD CHAIX}

Université de Tours, UMR 7323 